\title{
Hypercoagulation in COPD: the clot thickens
}

\author{
Farbod N. Rahaghi and Carrie L. Pistenmaa (1)
}

Pulmonary and Critical Care, Brigham and Women's Hospital, Harvard Medical School, Boston, MA, USA.

Corresponding author: Farbod Rahaghi (frahaghi@bwh.harvard.edu)

Copyright @The authors 2021

This version is distributed under the terms of the Creative Commons Attribution Non-Commercial Licence 4.0. For commercial reproduction rights and permissions contact permissions@ersnet.org

Received: 31 Aug 2021 Accepted: 18 Sept 2021

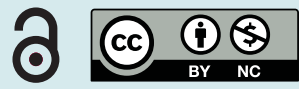

\author{
Shareable abstract (@ERSpublications) \\ There is a growing body of evidence that hypercoagulability is present in stable COPD, involves \\ changes in multiple coagulation factors, and is not simply associated with major causes of \\ inflammation and thrombosis https://bit.ly/3F5NnfN
}

Cite this article as: Rahaghi FN, Pistenmaa CL. Hypercoagulation in COPD: the clot thickens. ERJ Open Res 2021; 7: 00534-2021 [DOI: 10.1183/23120541.00534-2021].

A number of studies have found systemic measures of thrombosis to be elevated in both stable COPD and during times of exacerbation [1-5]. The data is strongest for plasma fibrinogen, a US Food and Drug Administration-approved biomarker for drug development in COPD that predicts exacerbations and mortality [6, 7]. While the results have been inconsistent for the many other coagulation biomarkers studied, the cumulative (published) evidence points to abnormalities in the coagulation cascade in COPD. However, those with COPD also have an increased risk of cardiovascular disease and elevated inflammatory markers [8,9], making these important potential confounders in any study of coagulation in COPD.

In this issue of ERJ Open Research, KyRIAKOPOulos et al. [10] add to the current data by presenting an analysis of a set of coagulation factors in a cohort of 103 patients with stable COPD and compare these with 42 control subjects. What makes this analysis compelling is the exclusion of participants with comorbid conditions associated with increased risk of thrombosis and inflammation, including any acute illness, heart failure, coronary artery disease, renal, liver or rheumatic disease, venous thrombosis, malignancy, or use of anticoagulant or antiplatelet drugs. Thus, their findings of elevated levels of coagulation factors (Factor II, V, VII, X, d-dimer and fibrinogen) and decreased levels of naturally occurring anticoagulants (protein $\mathrm{S}$ and antithrombin) in patients with stable COPD compared with smoking controls are in participants free of major confounding risk factors. They also confirmed these associations after excluding those with diabetes, another common cause of chronic inflammation and thrombosis [11]. Furthermore, they found a lower forced expiratory volume in $1 \mathrm{~s}$ to be associated with higher levels of Factor II, V and X, including after adjusting for age, body mass index, pack-years smoking history, current smoking status, inflammatory markers and low-density lipoprotein.

There are multiple ways in which a prothrombotic state may impact our understanding and treatment of patients with COPD. It is well known that comorbidities contribute significantly to the clinical decline and death of patients with COPD. A prothrombotic state may be linked to one or more of these comorbidities, as an increase in cardiovascular and cardiopulmonary diseases in COPD has been well described. This includes the presence of venous thromboembolism, particularly in the context of acute COPD exacerbations [12-16]. However, maintaining clinical vigilance for consequences of a prothrombotic state is not easy in patients with COPD. Many patients with advanced COPD, particularly those with frequent exacerbation, suffer from symptoms which include shortness of breath, hypoxaemia and chest tightness complicating the initial clinical suspicion for active coronary arterial disease or pulmonary thromboembolism. Interestingly, the current study suggests higher concentrations of Factors II, V and X with severity of obstruction, and those with more severe COPD have been found to also have a higher risk of venous thromboembolism as well [12]. While fibrinogen has been linked to risk of exacerbation and mortality in COPD, it may be worth further study of a panel of coagulation factors to identify those at highest risk of thrombotic disease who would most benefit from screening. 
Another potential way that a prothrombotic state may be related to COPD involves the pathogenesis and progression of COPD itself. The development and degree of airway obstruction, emphysema and exacerbation is quite heterogeneous in the face of the common insult of cigarette smoke. This suggests other harmful or protective factors are present, and known genetic factors still explain a minority of these differences [17]. One mechanistic hypothesis related to COPD progression includes endothelial cell activation and dysfunction [18, 19], which involves both inflammation and vascular injury with increased likelihood of coagulation. Platelet activation has also been found to be increased in stable COPD compared with smoking controls, in the setting of COPD exacerbation, and in those with hypoxaemia [20, 21]. There is additional observational data suggesting those taking aspirin have a slower progression of emphysema on computed tomography (CT), fewer COPD exacerbations and fewer respiratory symptoms [22, 23]. Lastly, there is evidence of a decreased therapeutic response to antiplatelet therapy in patients with COPD, which may be particularly relevant in treating comorbid cardiac diseases [24].

By focusing on stable COPD patients, this study largely controls for the use of glucocorticoids and differing severities of acute illness, which can themselves be contributors to the development of thromboembolism [25, 26]. However, the study does have some key limitations including a largely male population drawn from a single centre. Additionally, those with COPD had a median pack-years of smoking that was twice that of those without COPD, and thus the different smoking exposure remains a potential confounder in comparing the two groups [27]. While we acknowledge the importance of excluding major comorbid conditions with increased risk of thrombosis and inflammation, this also leads to concerns about the generalisability of the results as more than half of screened participants were excluded due to these risk factors: the study sample included only $36 \%$ of those screened with COPD and $43 \%$ of smoking controls.

Nonetheless, this paper adds to a growing body of evidence that hypercoagulability is present in stable COPD, involves changes in multiple coagulation factors, and is not simply associated with major causes of inflammation and thrombosis. We look forward to future studies on the relationship between coagulation, thrombosis and inflammation in COPD to further understand their role in exacerbations and disease progression, as well as helping us identify our patients at highest risk for thrombotic and cardiovascular complications.

Provenance: Commissioned article, peer reviewed.

Conflict of interest: F.N. Rahaghi has nothing to disclose. C.L. Pistenmaa reports grants from the NIH/NHLBI during the writing of this editorial; and grants from Boehringer Ingelheim, and personal fees from The Lancet Respiratory Medicine and CME, outside the submitted work.

References

1 Mkorombindo T, Dransfield MT. COPD: COagulation-associated Pulmonary Disease? Respirology 2021; 26: 290-291.

2 Ashitani J, Mukae H, Arimura Y, et al. Elevated plasma procoagulant and fibrinolytic markers in patients with chronic obstructive pulmonary disease. Intern Med 2002; 41: 181-185.

3 Husebø GR, Gabazza EC, D’Alessandro Gabazza C, et al. Coagulation markers as predictors for clinical events in COPD. Respirology 2021; 26: 342-351.

4 Liu M, Hu R, Jiang X, et al. Coagulation dysfunction in patients with AECOPD and its relation to infection and hypercapnia. J Clin Lab Anal 2021; 35: e23733.

5 Lankeit M, Held M. Incidence of venous thromboembolism in COPD: linking inflammation and thrombosis? Eur Respir J 2016; 47: 369-373.

6 Mannino DM, Tal-Singer R, Lomas DA, et al. Plasma fibrinogen as a biomarker for mortality and hospitalised exacerbations in people with COPD. Chronic Obstr Pulm Dis 2015; 2: 23-34.

7 Miller BE, Tal-Singer R, Rennard SI, et al. Plasma fibrinogen qualification as a drug development tool in chronic obstructive pulmonary disease. Perspective of the chronic obstructive pulmonary disease biomarker qualification consortium. Am J Respir Crit Care Med 2016; 193: 607-613.

8 Chen W, Thomas J, Sadatsafavi M, et al. Risk of cardiovascular comorbidity in patients with chronic obstructive pulmonary disease: a systematic review and meta-analysis. Lancet Respir Med 2015; 3: 631-639.

9 Karadag F, Karul AB, Cildag O, et al. Biomarkers of systemic inflammation in stable and exacerbation phases of COPD. Lung 2008; 186: 403-409.

10 Kyriakopoulos C, Chronis C, Papapetrou E, et al. Prothrombotic state in patients with stable COPD: an observational study. ERJ Open Res 2021; 7: 00297-2021.

11 Vazzana N, Ranalli P, Cuccurullo C, et al. Diabetes mellitus and thrombosis. Thromb Res 2012; 129: 371-377. 
12 Børvik T, Brækkan SK, Enga K, et al. COPD and risk of venous thromboembolism and mortality in a general population. Eur Respir J 2016; 47: 473-481.

13 Aleva FE, Voets L, Simons SO, et al. Prevalence and localization of pulmonary embolism in unexplained acute exacerbations of COPD: a systematic review and meta-analysis. Chest 2017; 151: 544-554.

14 Wedzicha JA, Hurst JR. Chronic obstructive pulmonary disease exacerbation and risk of pulmonary embolism. Thorax 2007; 62: 103-104.

15 Shapira-Rootman M, Beckerman M, Soimu U, et al. The prevalence of pulmonary embolism among patients suffering from acute exacerbations of chronic obstructive pulmonary disease. Emerg Radiol 2015; 22: 257-260.

16 Couturaud F, Bertoletti L, Pastre J, et al. Prevalence of pulmonary embolism among patients with COPD hospitalized with acutely worsening respiratory symptoms. JAMA 2021; 325: 59-68.

17 Busch R, Hobbs BD, Zhou J, et al. Genetic association and risk scores in a chronic obstructive pulmonary disease meta-analysis of 16,707 subjects. Am J Respir Cell Mol Biol 2017; 57: 35-46.

18 Malerba M, Nardin M, Radaeli A, et al. The potential role of endothelial dysfunction and platelet activation in the development of thrombotic risk in COPD patients. Expert Rev Hematol 2017; 10: 821-832.

19 Voelkel NF, Cool CD. Pulmonary vascular involvement in chronic obstructive pulmonary disease. Eur Respir J 2003; 22: Suppl. 46, 28s-32s.

20 Wedzicha JA, Syndercombe-Court D, Tan KC. Increased platelet aggregate formation in patients with chronic airflow obstruction and hypoxaemia. Thorax 1991; 46: 504-507.

21 Maclay JD, McAllister DA, Johnston S, et al. Increased platelet activation in patients with stable and acute exacerbation of COPD. Thorax 2011; 66: 769-774.

22 Aaron CP, Schwartz JE, Hoffman EA, et al. A longitudinal cohort study of aspirin use and progression of emphysema-like lung characteristics on CT imaging: the MESA lung study. Chest 2018; 154: 41-50.

23 Fawzy A, Putcha N, Aaron CP, et al. Aspirin use and respiratory morbidity in COPD: a propensity score-matched analysis in subpopulations and intermediate outcome measures in COPD study. Chest 2019; 155: 519-527.

24 Kunadian V, Wilson N, Stocken DD, et al. Antiplatelet therapy in the primary prevention of cardiovascular disease in patients with chronic obstructive pulmonary disease: a randomised controlled proof-of-concept trial. ERJ Open Res 2019; 5: 00110-2019.

25 Johannesdottir SA, Horváth-Puhó E, Dekkers OM, et al. Use of glucocorticoids and risk of venous thromboembolism: a nationwide population-based case-control study. JAMA Intern Med 2013; 173: 743-752.

26 Grimnes G, Isaksen T, Tichelaar Y, et al. Acute infection as a trigger for incident venous thromboembolism: results from a population-based case-crossover study. Res Pract Thromb Haemost 2018; 2: 85-92.

27 Tapson VF. The role of smoking in coagulation and thromboembolism in chronic obstructive pulmonary disease. Proc Am Thorac Soc 2005; 2: 71-77. 